REVIEW

\title{
Myocardial infarction in young adults
}

\author{
M Egred, G Viswanathan, G K Davis
}

Postgrad Med J 2005;81:741-745. doi: 10.1136/pgmj.2004.027532

Although myocardial infarction (MI) mainly occurs in patients older than 45 , young men or women can suffer Ml. Fortunately, its incidence is not common in patients younger than 45 years. However, the disease carries a significant morbidity, psychological effects, and financial constraints for the person and the family when it occurs at a young age. The causes of $\mathrm{Ml}$ among patients aged less than 45 can be divided into four groups: (1) atheromatous coronary artery disease; (2) non-atheromatous coronary artery disease; (2) hyper-coagulable states; (4) MI related to substance misuse. There is a considerable overlap between all the groups. This article reviews the literature and highlights the practical issues involved in the management of young adults with $\mathrm{Ml}$.

See end of article for authors' affiliations athors affilialions

Correspondence to: Dr M Egred, Cardiothoracic Centre, Thomas Drive, Liverpool L14 3PE, UK; m.egred@ ctc.nhs.uk

Submitted 3 August 2004 Accepted 22 January 2005

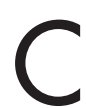
oronary heart disease (CHD) represents the leading cause of death in adults in the western world. ${ }^{12}$ Myocardial infarction (MI) is the lethal manifestation of CHD and can present as sudden death. Although MI mainly occurs in patients older than 45 , young men or women can suffer MI. Fortunately, its incidence is not common in patients younger than 45 years. $^{3}$ However, the disease carries a significant morbidity, psychological effects, and financial constraints for the person and the family when it occurs at a young age. The protection offered by young age has been slowly taken away by the increased prevalence of risk factors for CHD in adolescents such as smoking, obesity, and lack of physical activity. ${ }^{4}$

Better prognosis among young adults is achieved when the appropriate investigations and treatment are offered. Our interest in young adults with MI has been triggered by a series of patients presented with acute MI in their 20s and 30s after cocaine intake during Christmas and New Year period. The lack of published literature guiding physicians on the management of suspected MI in young patients aged less than 45 years has prompted us to a literature review to highlight the practical issues involved in their management.

The cut off age of 45 has been used in most studies to define young patients with CHD or MI and this same age will be used in this review.

\section{EPIDEMIOLOGY}

The incidence of CHD is declining in the UK in all was found to be $0.5 \%$ in men and $0.18 \%$ in women between 35 and 44 years, 20.5\% in men, and $17.1 \%$ in women over the age of 60 years. ${ }^{5}$ In age groups. The actual prevalence of the disease fact, the figures in young patients may be lower than actual because of atypical presentation and reluctance to submit themselves for further investigations. ${ }^{6}$ However, CHD in younger population aged less than 40 years was found to represent only $3 \%$ of all patients with $\mathrm{CHD}{ }^{7}$

The prevalence of risk factors is on the rise in young adults and children. This will result in an increased disease burden in the near future. Smoking, which has been traditionally recognised as the most common risk factor for heart disease, has been shown to be increasingly prevalent in young adults and adolescents reaching up to $9 \%$. In the UK, the smoking burden was found to be more among girls who also continued to stay as smokers for longer in their life. ${ }^{8}$ This would have an impact on the cardioprotection offered by hormones like oestrogen in young women. Obesity is a growing concern among young adults and children and it has increased by threefold in the UK in the past two decades. ${ }^{9-11}$ Insulin resistance, which by itself is a marker for CHD, has been found among $24 \%$ of school children in the USA. ${ }^{4}$ Metabolic syndrome and insulin resistance were found in two thirds of young people with MI. ${ }^{12}$ The disproportionate rise in prevalence of heart disease among certain ethnic groups like people of Asian Indian origin has been of great interest and these people tend to get MI at a younger age in addition to more complex coronary artery abnormalities. ${ }^{13} 14$

Cocaine use was found to be the commonest cause for the presentation of non-traumatic chest pain in the emergency department among young adults and can result in MI in the younger population. ${ }^{15}$ There is a fourfold increase in the number of cases in the past 10 years. In the UK, $45 \%$ of young adults have admitted use of recreational drugs, including cocaine, at least once. $^{16}$

It becomes clear that the prevalence of CHD is bound to rise in patients aged less than 45 in the years to come. The under reporting of the illness and the challenges involved in risk factors modification in this subset of patients would make the management of the potentially growing problem more difficult.

\section{PATHOGENESIS}

The causes for MI among patients aged less than 45 can be divided into four groups:

- Atheromatous CHD

- Non-atheromatous CHD

- Hypercoagulable states

Abbreviations: $\mathrm{MI}$, myocardial infarction; $\mathrm{CHD}$, coronary heart disease; $\mathrm{PCl}$, percutaneous coronary intervention; $C A B G$, coronary artery bypasss graft 
- MI related to substance misuse

There is a considerable overlap between all the groups. This classification is arbitrary but would guide the clinician towards appropriate management.

\section{ATHEROMATOUS CORONARY ARTERY DISEASE}

The atheromatous process starts in early childhood. In a necropsy study of 760 young adult patients who died of various causes, advanced CHD was found in $20 \%$ of men and $8 \%$ of women between the ages of 30 and 34 years of age. ${ }^{17}$ The pathological determinants of atherosclerosis in youth (PADY) study ${ }^{18}$ and Bogalusa heart study also reflect similar trends. ${ }^{19}$

The aetiology of atheromatous CHD was linked to the conventional risk factors as in adults. Among young patients with reported atheromatous process, cigarette smoking was found to be common in up to $92 \% .^{20}$ In a study of patients who had percutaneous coronary intervention (PCI), the prevalence of smoking was found to be higher in patients less than 40 years as compared with patients over 60 years $(58.7 \%$ and $43 \%, \mathrm{p}<0.01) .{ }^{21}$ In a study done in London among young patients with MI, positive family history of premature CHD was found in $39 \%$ of participants. ${ }^{22}$ The children born of parents with premature CHD tend to have more prevalence of lipid abnormalities, insulin resistance, and obesity strengthening the belief of a common genetic linkage. ${ }^{19}$ These people tend to have more arterial abnormalities than the rest of the patients who had MI younger than 45 years of age. ${ }^{23}$

Lipid abnormalities especially hypertriglyceridaemia and low HDL were found to be more common in patients who had their MI aged less than 45 years. Apart from overt diabetes, impaired glucose tolerance was found in $65 \%$ of survivors of MI aged less than 45 years. ${ }^{24}$

Conventional risk factors play a larger part in younger patients who had MI. Apart from these, emergence of novel risk factors for CHD like hyperhomocysteinaemia and lipoprotein (a) among adults of different age group may have the same clinical implications in younger people. ${ }^{25} 26$ Premature CHD is a rapidly progressive form of atheromatous process. ${ }^{22}$ Entirely unexplored areas like anger and psychosocial stress can add a significant morbidity and were associated with MI and coronary artery calcification in young adults. ${ }^{27}$

Angiographic findings significantly differed in patients less than 45 years. Increased prevalence of normal coronary arteries (up to $18 \%$ ) and minor coronary artery abnormalities were found in the CASS study. Single vessel disease was found in $38 \%$ of subjects. ${ }^{20}$ These findings highlight the role of vulnerable plaques and plaque rupture in younger people, similar to the older age groups, and the need to investigate the mechanisms that convert stable plaques into unstable plaques.

\section{NON-ATHEROMATOUS CORONARY ARTERY ABNORMALITIES}

Congenital coronary artery anomalies can present for the first time as MI in young adults. These are quite rare and may be a surprise to the cardiologist in the catheterisation laboratory. Cases of myocardial bridging, where the coronary arteries are embedded within a tunnel in the myocardium beneath a layer of muscles, have also been reported in young people presenting with MI. Myocardial bridging can result in significant ischaemia during systolic contraction and can result in MI. Both PCI and surgical splitting were found to be more useful than medical management in this group of patients. ${ }^{28-30}$
Coronary artery dissection can occur spontaneously in young adults. The chest pain is often atypical and women are at a higher risk during their peripartum period. The left anterior descending artery was found to be the culprit artery in most of the cases. Unfortunately the diagnosis is often made at necropsy. Successful treatment with stenting or surgery has been reported. ${ }^{31}{ }^{32}$

Septic vegetation from infected aortic valve was reported to have caused MI in younger people. Intravenous drug misusers are at a higher risk. ${ }^{33}$ MI has been reported to occur as a result of bacteraemia in the absence of vegetations in young people. ${ }^{34}$ The management usually entails treatment of the underlying source of sepsis as well. A rare cause of MI in younger adults is coronary artery aneurysms in which the mechanism of MI is thought to be either attributable to embolisation from the aneurysmal sac or extra luminal compression..$^{35}$ Paradoxical embolisation from right to left, through a patent foramen ovale, leading to MI has also been reported. ${ }^{3637}$

\section{RECREATIONAL DRUG USE}

Cocaine use is associated with various cardiac complications including MI. Among young patients admitted with nontraumatic chest pain in the emergency department, cocaine use was found to associate with the clinical presentation in $48 \%{ }^{38}$ A detailed history is vital as cocaine effects can present up to 76 hours after its use. Most of the patients who misuse cocaine are also found to be smokers and this makes them more vulnerable for MI.

MI was diagnosed, based on an increase in cardiac troponins, in $6 \%$ of the people admitted to the emergency department after various complications after cocaine use. Cocaine use results in acute MI by various mechanisms including coronary vasospasm and hypercoagulability in the background of heightened sympathetic activity. ${ }^{39}$ Long term cocaine use also results in hastened atherosclerosis. ${ }^{40}$ The diagnostic challenge in confirming MI in these people is complicated by the increased prevalence of false positive creatinine kinase rise. Serious arrhythmias including ventricular tachycardia can occur in cocaine users in the absence of MI. ${ }^{41}{ }^{42}$ Apart from MI, cocaine use has been associated with cardiomyopathy, tachyarrhythmias, and endocarditis. ${ }^{39}$

Amphetamine and marihuana use can result in MI but the data are limited. ${ }^{43}$ Binge drinking of alcohol has also been reported to be associated with developing MI in a young person, although the mechanism is not entirely clear. ${ }^{44}$

\section{HYPERCOAGULABLE STATES}

Antiphospholipid syndrome is associated with recurrent arterial and venous thrombosis. It is often the disease of the young in their 30s. It can be primary or secondary associated with other autoimmune diseases like systemic lupus erythematosus. Thrombotic occlusion of a coronary artery can result in acute MI. ${ }^{45}$ These patients tend to have premature atherosclerosis and increased platelet adhesiveness. ${ }^{46}{ }^{47}$ The titres of antiphospholipid antibodies need not be associated with disease activity and detailed evaluation is recommended to arrive at a diagnosis in suspected people. ${ }^{48}$

Nephrotic syndrome is associated with hypercoagulability attributable to the combination of various factors like disturbances of the fibrinolytic system, dyslipidaemia, and a decrease in anticoagulant factors. Reduction in concentration of antithrombin III, a coagulation inhibitor was particularly responsible for the thrombophilic tendency in most of the subjects. ${ }^{49}$

Factor V Leiden mutation is associated with a procoagulant state and has been reported to result in MI in young people; smokers are particularly at higher risk. ${ }^{50}$ Contraceptive pill 
use increases the risk of developing MI in young women because of its procoagulant activity. ${ }^{51}$

\section{CLINICAL PRESENTATION OF MI IN THE YOUNG PATIENT}

The clinical presentation of acute MI in young adults differs from their older counterparts. The classic presentation of worsening angina culminating in $\mathrm{MI}$ is rare in younger patients. The first onset of angina that rapidly progresses to fully evolved MI is often the case in patients less than 45 years of age. ${ }^{22}$ The prevalence of stable angina in young adults with reported coronary artery disease was only $24 \%$ in one study. ${ }^{22}$ In a series of patients who had their MI less than 45 years of age, $69 \%$ denied any chest pain before MI. The duration of symptoms was found to be less than a week in most of the patients. ${ }^{52}$ Careful history taking would give important clues as to the differential diagnosis of chest pain in young adults such as myopericarditis and pulmonary embolism.

In every young patient presenting with MI, use of recreational drugs in the recent times should be recorded. Family history of premature CHD, risk factor profile such as smoking, obesity, diabetes, and dyslipidaemia would give better clues as to the likelihood of atheromatous coronary artery disease. History of recurrent venous and arterial thrombosis should also be reported.

Initial clinical examination should concentrate on heamodynamic stability, evidence of sympathetic hyperactivity such as tachycardia and sweating, and evidence of previous injected drug misuse. Stigmata of dyslipidaemia such as xanthelasma, arcus senilis, and tendon xanthomata should be looked for in every person. Upon investigations, ST segment elevation in the ECG is often noted if the patient presents to the emergency department soon after the onset of chest pain. The value of serial ECGs should never be underestimated, as dynamic ST segment elevation in the ECG is associated with cocaine use. Both the chest pain and ECG changes are promptly found to return to normal after administration of vasodilators in patients with coronary artery spasm related to cocaine use. ${ }^{39}$ Abnormal Q waves are usually seen in patients who present later than 12 hours after the onset of chest pain. Non-specific T wave changes, ST depression, and $\mathrm{T}$ wave inversion are seen in patients with partially occluded coronary arteries. The presence of pleuritic type chest pain and concave upward ST segment elevation in lateral leads would suggest the possibility of myopericarditis. $^{53}$

Cardiac enzymes are invariably raised in all the people with MI. Cardiac specific troponin $\mathrm{T}$ increase is considered to be the most sensitive marker of myocardial damage. False positive creatinine kinase increase is seen in patients who misuse cocaine. ${ }^{42}$

\section{MANAGEMENT}

Initial management of MI in younger patients differs slightly from the standard management in adults. Initial administration of oxygen, diamorphine, nitrates, and aspirin should be considered in all patients. In patients with history of cocaine use, $\beta$ blockers should be delayed for 48 hours as unopposed $\alpha$ stimulation may result in severe coronary spasm and paradoxical worsening of chest pain. Benzodiazipines are recommended in the initial management of MI in cocaine misuser. Nitrates should be continued in these people to counteract coronary spasm. ${ }^{39}$ In patients with haemodynamic instability specialist advice should be sought early and the scope for early coronary angiography and intervention should be assessed.

Patients with cocaine induced persistent ST elevation not resolved by nitrates, should be offered thrombolytic therapy.
Younger patients seem to tolerate the thrombolytic agents better with a good ST segment resolution in the ECG. ${ }^{54}$ In patients with non-ST segment elevation MI, the initial management should be followed by risk stratification based on persistent or dynamic ECG changes, higher degree of cardiac enzyme rise, and presence of other risk factors like diabetes mellitus. Higher risk patients should be referred to the specialists to assess the need for early coronary angiography and intervention. Coronary angiography is not routinely offered in all the younger patients as a significant number tend to have normal coronary arteries.

In patients with established MI, exercise stress testing could well be a useful tool for risk stratification. Most of the younger patients who managed stage 3 of the Bruce protocol (nine minutes or more) were found to have normal coronary arteries. ${ }^{55}$ Echocardiographic assessment of left ventricular function should be done in all patients. ${ }^{52}$

\section{REVASCULARISATION}

Coronary angiography is generally performed in most of the patients with MI. As discussed previously this probably may not be offered as a routine choice in all the affected patients because of increased chances of finding a normal coronary artery. In patients with risk factors such as diabetes mellitus, dyslipidaemia, and family history of premature CHD, the probability of finding abnormal coronary artery is higher. ${ }^{20} 56$ Coronary angiography should be offered to people with significant left ventricular dysfunction as this group of patients has a better outcome with early revascularisation..$^{52}$

In a series of 843 patients with reported coronary artery disease, the prognosis was favourable in the group of patients who received revascularisation treatments like PCI and coronary artery bypass grafting $(\mathrm{CABG}) .^{55}$ PCI in younger patients had fewer post-procedural complications compared with MI rate of $5 \%$, urgent CABG of $1 \%$, and mortality of $1 \%$ in the older patients. Elective CABG was needed in 5\% (four patients) in young patients over a three years period. ${ }^{57}$ The success rate of percutaneous procedures was high in younger patients approaching $93 \% .^{21}$ In the current era of drug eluting stents and glycoprotein IIb-IIIa inhibitors the success rates are expected to be higher and long term studies are awaited.

CABG should be offered to patients with triple vessel disease, complex coronary artery abnormalities, and impaired left ventricular function. CABG carries a better success rate in younger patients. Survival rates were found to be $92 \%$ at five years and $86 \%$ in 10 years respectively in patients less than 40 years, ${ }^{58}$ compared with $75 \%$ and $58 \%$ for patients over 65 years old. ${ }^{59}$ Arterial grafts are preferred over venous grafts because of better long term patency. ${ }^{58}$

\section{SECONDARY PREVENTION}

MI in younger patients does carry a better prognosis if appropriately treated. On the other hand, poor control of risk factors carries a significant morbidity and mortality. ${ }^{21}$ Antiplatelet agents like aspirin and clopidogrel should be used as per the guidelines for adults. Warfarin is necessary in patients in a hypercoaguable state and continued lifelong in patients with recurrent ischaemic events. ${ }^{60}$ Start of $\beta$ blockers can be delayed for a few days as they may worsen coronary spasm.

Statins are invariably prescribed in all patients with MI and their clinical effects extend beyond lipid lowering. Statins are said to stabilise plaques in patients with atheromatous CHD, thereby improving their outcome, and reducing recurrent events. Other agents like niacin and omega 3 fatty acids should be considered in special situations like hypertriglyceridaemia and low HDL concentrations. ${ }^{61}{ }^{62} \mathrm{~B}$-complex vitamins are useful in patients with hyperhomocysteinaemia. ${ }^{63}$ 
Angiotensin converting enzyme inhibitors (ACE-I) should be offered to all patients with left ventricular dysfunction as substantial benefits were shown in using ACE-I in this group of patients. ${ }^{6465}$

Lifestyle changes play an important part in the management of these patients. Stopping smoking should be strongly advised. Extensive progression of CHD was noted in younger patients who continue to smoke after their bypass surgery. Good control of diabetes and correction of lipid abnormalities were shown to improve prognosis in patients less than 45 years. ${ }^{66}$ Risk factors modification could prove to be a challenging task in these people.

In general as discussed earlier, younger patients carry a better prognosis in the earlier period after their MI than older patients. In contrast with popular belief, Cole et al have found that the mortality in young patients with MI was as high as $30 \%$ at 15 year follow up..$^{55}$ Part of this high mortality may be attributable to inclusion of a significant number of patients with diabetes mellitus and ejection fraction less than $30 \%$. Better prognosis was found in patients who had single vessel coronary artery disease. ${ }^{66}{ }^{67}$ Revascularisation was associated with better success rate and survival, hazards ratio of 0.51 for PCI and 0.68 for CABG in patients aged less than 45 years. ${ }^{55}$

\section{CONCLUSION}

Although MI, fortunately, is an uncommon entity in young adults aged less than 45 years, it constitutes an important problem for both the patient and the treating physician. It has a devastating effect on the more active lifestyle of young patients. These young patients also have a different risk factor profile, clinical presentation, and prognosis in comparison with older patients, which has to be taken into consideration when treating these young adults presenting with MI. The increased prevalence of risk factors for CHD may set up an alarming trend.

Substance misuse, coronary artery anomalies, premature coronary artery disease, and hypercoagulable state have to be considered in all patients with suspected MI who are less than 45 years. Early stabilisation should be followed by risk stratification, and early revascularisation, where appropriate, should be offered as it carries a better clinical outcome. Risk factors modification should be emphasised.

We strongly emphasise the importance of secondary preventive measures in all young patients admitted with MI as the long term mortality can reach up to one third if not treated appropriately.

\section{Authors' affiliations \\ M Egred, Cardiothoracic Centre, Liverpool, UK \\ G Viswanathan, G K Davis, Aintree Cardiac Centre, University Hospital Aintree, Liverpool, UK \\ Funding: none. \\ Conflicts of interest: none.}

\section{REFERENCES}

1 Department of Health. National Service Framework for Coronary Heart Disease, 2000. http://www.doh.gov.uk/publications.

2 Office of National Statistics. Indicators of the nation's health-ischaemic heart disease: male and female death rates by special causes, 2002. http:// www.statistics.gov.uk/mortality.

3 Office of National Statistics. Weekly incidence of heart attacks http:// www.statistics.gov.uk/morbidity/cardiovascular diseases.

4 Sinha R. Fisch G, Teague B, et al. Prevalence of impaired glucose tolerance among children and adolescents with marked obesity. N Engl J Med 2002:346:802-10.

5 Office of National Statistics. Key health statistics from General Office, 2000. http://www.statistics.gov.uk/health and care.

6 Klein LW, Nathan S. Coronary artery disease in young adults. J Am Coll Cardiol 2003;41:529-31.

7 Jaloweil DA, Hill JA. Myocardial infarction in young men and women. Cardiovasc Clin 1989;20:197-206.
8 Department of Health. Drug use, smoking and drinking among young people in England in 2001. http://www.doh.gov.uk/publication/publication statistics.

9 British Heart Foundation. BHF coronary heart disease statistics 2003. http:// www.bhf.org.uk/professionals/statistics.

10 Ebbeling CB, Pawlak BB, Ludwig DS. Lancet 2002;360:473-82

11 Horton R. Who pays in the obesity war? Lancet 2003;363:339.

12 Curren PJ, Chung EH, Chauhan MS. Metabolic syndrome: an under recognized risk factor for myocardial infarction in the young. (Abstract). J Am Coll Cardiol 2004;43:249A.

13 Bhatnagar D, Anand IS, Durrington PN, et al. Coronary risk factors in people from Indian sub-continent living in West London and their siblings in India. Lancet 1995:345:404-9.

14 Enas EA, Mehta JL. Malignant coronary artery disease in young Asian Indians: thoughts on pathogenesis, prevention and treatment. Clin Cardiol 1995; 18:131-5

15 Jay SJ, Hollander JE. Cocaine use and chest pain syndromes. Arch Intern Med 1998; 158:1827-8

16 Office of National statistics. Drug abuse, misuse of alcohol and smoking. http://www. statistics.gov.uk/cci.

17 McGill HC Jr, McMahan CA, Zieske AW, et al. Association of coronary heart disease risk factors with microscopic qualities of coronary atherosclerosis in youth. Circulation 2000;102:374.

18 Milonig G, Malcolm GT, Wick G. Early inflammatory and immunological lesions in juvenile atherosclerosis from the pathological determinants of atherosclerosis in youth (PDAY) study. Atherosclerosis 2002;160:444-8.

19 Berenson GS, Srinivasan SR, Bao W, et al. Association between multiple cardiovascular risk factors and atherosclerosis in children and young adults: the Bogalusa heart study. N Engl J Med 1998;338:1650-6.

20 Zimmerman FH, Cameron A, Fisher LD, et al. Myocardial infarction in young adults: Angiographic characteristics, risk factors and prognosis, coronary artery surgery study register (CASS). J Am Coll Cardiol 1995;26:654.

21 Mukherjee D, Hsu A, Moliterno DJ, et al. Risk factors for premature coronary artery disease and determinants of adverse outcomes after revascularisation in patients less than 40 years old. Am J Cardiol 2003;92:1465-7.

22 Chen L, Chester M, Kaski JC. Clinical factors and angiographic features associated with premature coronary artery disease. Chest 1995; 108:364.

23 Gaeta G, de Michale M, Cuomo S, et al. Arterial abnormalities in the offspring of patients with premature myocardial infarction. N Engl J Med 2000;343:840-6.

24 Malmberg K, Barenholm P, Hamsten A. Clinical and biochemical factors associated with prognosis after myocardial infarction at a younger age. J Am Coll Cardiol 1994;24:592-4.

25 Foody JM, Milberg JA, Robinson K, et al. Homocysteine and lipoprotein (a) interact to increase coronary artery disease risk in young men and women. Arteriosclerosis Throm Vasc Biol 2000;20:493-9.

26 Issar HS, Puri VK, Narain VS, et al. Lipoprotein (a) and lipid levels in young patients with myocardial infarction and in their first degree relatives. Indian Heart J 2001;53:463-6.

27 Iribarren C, Sydney S, Bild DE, et al. Association of hostility with coronary artery calcification in young adults; the CARDIA study. Coronary artery risk determinants in young adults. JAMA 2000;283:2546-51.

28 Klues HG, Schwartz ER, Vom Dahl S, et al. Disturbed intra coronary heamodynamics myocardial bridging: early normalisation by intra coronary stent placement. Circulation 1997;96:2905-13.

29 Stables RH, Knight CJ, McNeill JG, et al. Coronary stenting in the management of myocardial ischaemia caused by muscle bridging. $\mathrm{Br}$ Heart $\mathrm{J}$ 1995:74:90-2.

30 Möhlenkamp S, Hort W, Ge J, et al. Update on myocardial bridging. Circulation 2006; 106:2616.

31 Vale PR, Baron DW. Coronary artery stenting for spontaneous coronary artery dissection: a case report and review of literature. Cathet Cardiovas Diagn 1998;45:280-6.

32 Butler R, Webster MWI, Davies GK, et al. Spontaneous dissection of native coronary arteries. Heart 2005;91:223-4.

33 Mathew J, Addai T, Anand A. Clinical features, site of involvement, bacteriological findings and outcomes of infective endocarditis in intra venous drug users. Arch Intern Med 1995; 155:1641-6.

34 Valtonen V, Kuikka A, Syrjanen J. Thromboembolic complication in bacteremic infections Eur Heart J 1993;14(suppl K):20-3.

35 Dagalp Z, Pamir G, Alpman A, et al. Coronary artery aneurysms. Report of two cases and review of literature. Angiology 1996;47:197-201.

36 Egred M, Patel JC, Walton S. Impending paradoxical embolism. Circulation 2001; 103:113-14e.

37 Egred M, Patel JC, Walton S. Management of paradoxical coronary embolism. Circulation 2001;104:153e.

38 Hollander JE, Todd KH, Green G, et al. Chest pain associated with cocaine: an assessment of prevalence in suburban and urban emergency departments. Ann Emerg Med 1995;26:671-6.

39 Lange RA, Hills CD. Cardiovascular complications of cocaine use. NEngl J Med $2001 \cdot 345: 351-8$.

40 Kolodgie KD, Virmani R, Cornhill JF, et al. Increase in atherosclerosis and adventitial cell mass in cocaine abusers - an alternative mechanism of cocaine associated coronary vosospasm and thrombosis. J Am Coll Cardiol $1991 ; 17: 1553-60$.

41 Gitter MJ, Goldsmith SR, Duntar DN, et al. Cocaine and chest pain: clinical features and outcomes of the patients hospitalised to rule out myocardial infarction. Ann Intern Med 1991; 1 15:277-82.

42 Hollander JE, Levitt MA, Young GP, et al. Effect of recent cocaine use on the specificity of cardiac markers for diagnosis of acute myocardial infarction. Am Heart J 1998; 135:245-52. 
43 Ghuran A, van Der Wieken LR, Nolan J. Cardiovascular complications of recreational drugs. BMJ 2001;323:464-6.

44 Gowda RM, Khan IA, Vasavada BC, et al. Alcohol-triggered acute myocardial infarction. Am J Ther 2003;10:71-2.

45 Jouhikainen T, Pohjola-Sintonen S, Stephansson E. Lupus anticoagulant and cardiac manifestation in systemic lupus erythematosus. Lupus 1994;3:167.

46 Harats D, George J, Levy Y, et al. Atheroma links with an antiphospholipid antibodies: Hughes syndrome and lupus. Q J Med 1999;92:57-9.

47 Vaarala $O$. Antiphospholipid antibodies and athrtrosclerosis. Lupus 1996;5:442-7.

48 Quismorio FP Jr. Clinical application of serological abnormalities in systemic lupus erythematoses. In: Wallace DJ, Hahn BH, eds. Dubois' lupus erythematoses. 5th ed. Baltimore: William and Wilkins, 1997:925-42.

49 Osula S, Bell GM, Hornung RS. Acute myocardial infarction in young adults: causes and management. Postgrad Med J 2002;78:27-31.

50 Tanis BC, Bloemankamp DG, Van Den Bosch MA, et al. Prothrombotic coagulation defects and cardiovascular risk factors in young women with acute myocardial infarction. Br J Haematol 2003;1:471.

51 Petitti DB. Combination estrogen-progestin oral contraceptives. N Engl J Med 2003;349: 1443-50.

52 Klein LW, Agarwal JB, Herlich MB, et al. Prognosis of symptomatic coronary artery disease in young adults aged 40 years or less. Am J Cardiol 1987;60:1269-72.

53 Schamroth L. Pericarditis. In: An introduction to electro cardiography. 7th ed. Oxford: Oxford University Press, 1990:189-95.

54 Chouhan L, Hajan HA, Pomposiello JC. Comparision of thrombolytics therapy for acute myocardial infarction in patients aged less than 35 and, more than 55 years. Am J Cardiol 1993;71:157.

55 Cole JH, Miller III JI, Sperling LS, et al. Long-term follow-up of coronary artery disease presenting in young adults. J Am Coll Cardiol 2003;41:521-8.

56 Fournier JA, Sanchez A, Quero J, et al. Myocardial infarction in men aged 40 years or less: a prospective clinical angiographic study. Clin Cardiol 1996;19:531-6.
57 Mehan VK, Urben P, Dornez PA, et al. Coronary angioplasty in the youngprocedural results and later outcome. J Invasive Cardiol 1994;6:202.

$58 \mathrm{Ng}$ WK, Vedder M, Whitlock RM, et al. Coronary revascularisation in young adults. Eur J Cardiothorac Surg 1997;11:732.

59 Floten HS, Ahmed A, Swanson JS, et al. Long term survival after post infarction bypass operation, early and late operation. Ann Thorac Surg 1989;48:757.

60 Schafer Al, Levine MN, Konkle BA, et al. Thrombotic disorders: diagnosis and treatment. Hematology 2003;520-39.

61 Miller M. Niacin as a component of combination therapy for dyslipidaemia. Mayo Clin Proc 2003;78:735-42.

62 Din JN, Newby DE, Flapan AD. Omega 3 fatty acids and cardiovascular disease-fishing for a natural treatment. BMJ 2004;328:30-5.

63 Welch GN, Loscalzo J. Homocysteine and atherothrombosis. N Engl J Med 1998;338:1042-50.

64 The Heart Outcomes Prevention Evaluation study investigators Effects of angiotensin converting enzyme inhibitor ramipril on cardiovascular events in high-risk patients. N Engl J Med 2000;342:145-53.

65 The EUROpean trial on reduction of cardiac events with perindopril in stable coronary artery disease envestigators. Efficacy of perindopril in reduction of cardiovascular events among patients with stable coronary artery disease: randomised double blind, placebo controlled, multicentre trial (the EUROPA study). Lancet 2003;362:782-8.

66 Moccetti T, Malacrida R, Pasotti E, et al. Epidemiologic variables and outcome of 1972 young patients with acute myocardial infarction. Data from GISSI-2 database. Investigators of the Gruppo Italiano per lo Studio della Sopravvienza nell'Infarto Miocardico (GISSI-2). Arch Intern Med 1997; 157:865

67 Porter A, Wurzel M, Ben-Gal T, et al. Long term prognosis of 210 patients who underwent coronary angiography before 40 years of age. Am J Cardiol 1998;81:1168. 